the ratio $a_{1} / a_{2}$, and by suitably assigning the value of this ratio the surface condition can be satisfied whatever $p_{1}$ and $p_{8}$ may be.

Hence, in this case, $p_{1}$ and $p_{2}$ may be any two negative quantities satisfying the relation

$$
p_{1}+p_{8}=-m^{\prime}
$$

and the velocity of propagation is given by

$$
V^{2}=\frac{g m^{\prime}}{p_{1} p_{2}+m^{2}}
$$

For a given value of $m$, the greatest of these values of $\nabla^{2}$ is $\mathrm{gm}^{\prime} / \mathrm{m}^{2}$, so that the irrotational mode would seem to stand by itself, and not to occur as one limiting case of the rotational modes.

It might have been expected, $\grave{a}$ prior $i$, that in the case of infinite depth, the infinity of possible motions would have been of a higher order than in the case of finite depth; and this is seen to be so, the former case being comparable with the infinity of possible positions of points on a line, and the latter vith the infinite series of roots of a trunscendental equation.

On some Rings of Circles connected with a Triangle, and the Circles which cut them at Equal Angles. By W. W. TAYLOR.

[Rend June 13th, 1889.]

If any three circles be placed in contact, the lines joining their points of contact $A, B, C$ form a triangle. Hence it would appear that such three circles must play an important part in the geometry of the triangle. They mas be defined, with reference to the triangle $A B C$, as the circles* that touch two of the radii of the circle $A B C$ at the angular points of the triangle. We will proceed to find their equations, and discuss their properties, and those of certain associated circles and triangles.

- These circles have been ablled the ex-cosine circles of the triangle $A B C$. (W. E. Johnson's "Trigonometry," \$ 194.) 
$398 \mathrm{Mr}$. W. W. Taylor on some Rings of Circles, \&c. [June 13,

[The centres of the same set of circles form a second triangle, and the circles may be defined, with reference to that triangle, as the circles which are orthogonal to the inscribed circle. and have their centres at the angular points of the triangle. The properties obtained for the first set may he transformed so as to suit the second set of circles by means of three formulo like

$$
\frac{\delta}{d}=\frac{R}{r s}\left(-a \cos ^{2} \frac{A}{2}+\beta \cos ^{8} \frac{B}{2}+\gamma \cos ^{2} \frac{C}{2}\right),
$$

where $\alpha, \beta, \gamma$ are the trilinear coordinates of a point reforred to the first triangle $A B C$, and $\delta, \epsilon, \xi$ new coordinates referred to the sides $d, e, f$, i.e., $E F, F D, D E$, where $D, E, F$ are the feet of the perpendiculars from $A, B, C$ on the opposite sides.]

If the equation

$$
\begin{gathered}
\beta \gamma \sin A+\gamma \alpha \sin B+\alpha \beta \sin O \\
-(l a+m \beta+\imath \gamma)(\alpha \sin A+\beta \sin B+\gamma \sin C)=0
\end{gathered}
$$

represent a circle orthogonal to the circle $A B C$, the straight line whose eqnation is $l \alpha+m \beta+u \gamma=0$ must be the polar of $O$ the centre of the circle $A B C$, i.e., of the point $R \cos \dot{A}, \pi \cos B, R \cos O$. The condition for this is

$$
1-l \cos A-m \cos B-n \cos C=0
$$

and the equation of the circle becomes

$$
\begin{gathered}
(l \cos A+m \cos B+n \cos C)(\beta \gamma \sin A+\gamma \alpha \sin B+\alpha \beta \sin C) \\
-(l \alpha+m \beta+n \gamma)(\alpha \sin A+\beta \sin B+\gamma \sin C)=0,
\end{gathered}
$$

which can be written in the form

$$
\begin{gathered}
l \sin A\left(\alpha^{2}-\beta \gamma \cos A+\gamma \alpha \cos B+a \beta \cos C\right) \\
+m \sin B\left(\beta^{3}+\beta \gamma \cos A-\gamma \alpha \cos B+\alpha \beta \cos C\right) \\
+n \sin O\left(\gamma^{2}+\beta \gamma \cos A+\gamma \alpha \cos B-\alpha \beta \cos C\right)=0 .
\end{gathered}
$$

If the common chord be the line $a=0$, then $l=+\sec A$, and the equation of the circle orthogonal to the circle $A B C$ is

$$
a^{2}-\beta \gamma \cos A+\gamma a \cos B+\alpha \beta \cos C=0 \text {. }
$$

We will denote the left-hand side of this by the letter $A_{1}$; the equations of the three circles that touch $O B, O C ; O C, O A ; O A, O B$ at the angular points will be

$$
\left.\begin{array}{l}
A_{1} \equiv \alpha^{2}-\beta \gamma \cos A+\gamma \alpha \cos B+\alpha \beta \cos O=0 \\
I_{1} \equiv \beta^{2}+\beta \gamma \cos A-\gamma u \cos B+\alpha \beta \cos C=0 \\
C_{1} \equiv \gamma^{2}+\beta \gamma \cos A+\gamma a \cos B-\alpha \beta \cos C=0
\end{array}\right\}
$$


The equation of any other circle orthogonal to the circle $A B O$ will be $l \sin A \cdot A_{1}+m \sin B \cdot B_{1}+n \sin C \cdot O_{1}=0$

where $l a+m \beta+n \gamma=0$ is the equation of their common chord.

It will be observed that, as each of the three circles $A_{1}, B_{1}, C_{2}$ touches the other two, and as there must be a pair of circles that each touches, these three circles, $A_{1}, B_{1}, C_{2}$, must form a ring in the sense of Mr. H. M. Taylor's paper on "The Porism of the Ring of Circles touching Two Circles," Messenger of Mrathematics, Vol. vir., 1878.)"

Wo shall accordingly refer to them as the 3-ring circles $A_{1}, B_{1}, C_{2}$.

The centre of the circle $A_{1}$ is most easily found by taking the tangents to the circumscribed circlo at $B, C$, und finding their intersection.

It is obviously

$$
\frac{a}{-a}=\frac{\beta}{b}=\frac{\gamma}{c}=\frac{2 \Delta}{-a^{2}+b^{2}+c^{2}}
$$

Henco we see that the centres of these circles are the "associates of the Lemoine point."

'l'o find the length of the intercept cut off on the side $A O$ of the trianglo by the circle $A_{1}$, we have the equations

$$
\beta=0, \quad a=-\gamma \cos B \text { by (3), and } a \alpha+c \gamma=2 \Delta \text {, }
$$

wlience

$$
a=-a \sin C \cos B \sec A \text {, }
$$

and the intercept

$$
C P= \pm a \operatorname{cosec} C= \pm a \cos B \mathrm{sec} A ;
$$

similarly the intorcept

$$
B Q= \pm a \cos C \sec A,
$$

alil

$$
B C: C P: B Q=\cos A: \pm \cos B: \pm \cos C \text {. }
$$

It is worthy of notice thiat the six points conesponding to $P$, Q lie on the conic

$$
\Sigma a^{2}+\Sigma i \beta \gamma(\cos A+\sec A)=0,
$$

and also that

$$
A_{1} B_{1}+B_{1} C_{1}+C_{1} A_{1} \equiv\{\Sigma(\beta \gamma \sin A)\}^{8}
$$

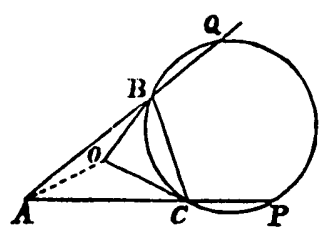

"Compare also a paper in the samo volume "On the Ring of Circles touching Two Circles, and kindred Porisms." 
400 Mr. W. W. Taylor on some Rings of Circles, \&c. [June 13,

\section{LEMMA I.}

If $a, \beta, \gamma$ be the trilinear coordinates of a point, the equation of a circle being expressed in the form

$$
\phi(a, \beta, \gamma) \equiv \Sigma \beta \gamma \sin A-(l a+m \beta+n \gamma) \Sigma a \sin A=0,
$$

or in the form

$$
(l, m, n) \equiv \Sigma a \beta \gamma-(l a+m \beta+n \gamma) \Sigma a a=0,
$$

the coordinates of its centre are $\bar{\alpha}, \bar{\beta}, \bar{\gamma}$, where

$$
\begin{aligned}
& \bar{a}=R(-l+m \cos C+n \cos B+\cos A), \\
& \bar{\beta}=R(-m+n \cos A+l \cos C+\cos B), \\
& \bar{\gamma}=R(-n+l \cos B+m \cos A+\cos C),
\end{aligned}
$$

and its radius is $\rho$, where

$\rho^{2}=R^{2}\left(l^{2}+m^{2}+n^{2}-2 m n \cos A-2 n l \cos B-2 l m \cos C\right.$

$$
-2 l \cos A-2 m \cos B-2 n \cos C+1) \text {. }
$$

The condition that the centre of the circle $(l, m, n)$ is the pole of the line at infinity gives the following equations to find its coordinates :-

$$
\begin{aligned}
& \{c \beta+b \gamma-a(l a+m \beta+n \gamma)-l(a a+b \beta+c \gamma)\} / a \\
= & \{a \gamma+c a-b(l a+m \beta+n \gamma)-m(a \alpha+b \beta+c \gamma)\} / b \\
= & \{b a+a \beta-c(l a+m \beta+n \gamma)-n(a \alpha+b \beta+c \gamma)\} / c .
\end{aligned}
$$

Let each of these $=X-(l a+m \beta+n y)$. Then we have the equations

$$
\begin{aligned}
& c \beta+b \gamma-l(a a+b \beta+c \gamma)=a X, \\
& a \gamma+c a-m(a \alpha+b \beta+c \gamma)=b X, \\
& b a+a \beta-n(a \alpha+b \beta+c \gamma)=c X .
\end{aligned}
$$

Then, eliminating $\beta, \gamma$, we obtain the equation

$$
a\left|\begin{array}{ccc}
-a l & c-b l & b-c l \\
c-a m & -b m & a-c m \\
b-a n & a-b n & -c n
\end{array}\right|=X\left|\begin{array}{ccc}
a & c-b l & b-c l \\
b & -b i n & a-c m \\
c & a-b n & -c n
\end{array}\right|
$$


1889.] Mr. W. W. Taylor on some Rings of Circles, \&c.

The coefficient of $X$ becomes, on expansion,

$2 a b c(\cos A-l+m \cos C+n \cos B)$

Therefore we have

$$
\boldsymbol{a}: \boldsymbol{\beta}: \boldsymbol{\gamma}: a \boldsymbol{\alpha}+b \boldsymbol{\beta}+c \boldsymbol{\gamma}
$$

$=\cos A-l+m \cos C+n \cos B: \cos B-m+n \cos A+l \cos C$

$: \cos C-n+l \cos B+m \cos A: a \cos A+b \cos B+c \cos C$;

but

$$
a a+b \beta+c \gamma=a R \cos A+b R \cos B+c R \cos C,
$$

where $R$ is the radius of the circle $A B C$;

therefore

$$
\left.\begin{array}{l}
\boldsymbol{a}=R(\cos A-l+m \cos C+n \cos B) \\
\beta=R(\cos B-m+n \cos A+l \cos C) \\
\gamma=R(\cos C-n+l \cos B+m \cos A)
\end{array}\right\}
$$

and, if $\rho$ is the radius of the circle $\phi=0$,

$$
\rho^{2} \phi(-1, \cos C, \cos B)=-\phi\left(\alpha_{0}, \beta_{0}, \gamma_{0}\right) ;
$$

whence, by substituting the above values, we obtain

$\rho^{2}=R^{2}\left(l^{2}+m^{2}+n^{2}-2 m n \cos A-2 n l \cos B-2 l m \cos C\right.$

$$
-2 l \cos A-2 m \cos B-2 n \cos C+1)
$$

\section{LemMa II.}

We will now proceed to find the cosine of the angle $\theta$, at which two circles $\left(l_{1}, m_{1}, n_{1}\right),\left(l_{2}, m_{2}, n_{2}\right)$ whose equations are expressed in the form (1) cut one another.

If the centres of these circles be $O_{1}, O_{2}$, and their radii $R_{1}, R_{2}$,

$$
\cos \theta=\frac{O_{1} O_{2}^{2}-R_{1}^{2}-R_{2}^{2}}{2 R_{1} R_{2}}
$$

and

$$
R_{1}=\frac{8 R^{3}}{a b c} \phi_{1}
$$

where $\phi_{1}$ denotes the value of the expression

$$
\Sigma, \beta \gamma \sin A-(\Sigma / \alpha)(\Sigma a \sin A),
$$

when for $a, \beta, \gamma$ we substitute the coordinates of the centre.

Let the equations of the circles whose radii are $R_{1}, R_{2}$, and centres $O_{1}, O_{\eta}$, be

$$
\begin{array}{r}
\phi_{1}(\alpha, \beta, \gamma) \equiv \Sigma \beta \gamma \sin A-\left(\Sigma l_{1} a\right)(\Sigma a \sin A), \\
\phi_{\Omega}(a, \beta, \gamma) \equiv \Sigma \beta \gamma \sin A-\left(\Sigma l_{q} a\right)(\Sigma a \sin A) . \\
- \text { No. 368. } 2 \dot{\mathrm{D}}
\end{array}
$$

voL. XX.—NO. 368 . 
402 Mr. W. W. Taylor on some Rings of Circles, \&c. [June 13, Then the equation of a circle whose centre is $O_{1}$ and radius $O_{1} O_{1}$, will be

$$
\phi_{1}(\alpha, \beta, \gamma)+h(\Sigma \alpha \sin A)^{9}=0 ;
$$

and, as $O_{2}\left(\alpha_{3}, \beta_{3}, \gamma_{3}\right)$ is on this circle,

$$
\phi_{1}\left(a_{2}, \beta_{2}, \gamma_{2}\right)+h(\Sigma a \sin A)^{2}=0 ;
$$

and

$$
\begin{aligned}
O_{1} O_{2}^{2} & =\frac{\delta R^{3}}{a b c}\left\{\phi_{1}\left(a_{1}, \beta_{1}, \gamma_{1}\right)+h(\Sigma a \sin A)^{3}\right\} \\
& =\frac{8 \pi^{3}}{a b c}\left\{\phi_{1}\left(\alpha_{1}, \beta_{1}, \gamma_{1}\right)-\phi_{1}\left(\alpha_{2}, \beta_{2}, \gamma_{2}\right)\right\},
\end{aligned}
$$

and

$$
\begin{aligned}
\cos \theta & =\frac{\phi_{1}\left(a_{1}, \beta_{1}, \gamma_{1}\right)-\phi_{1}\left(a_{3}, \beta_{2}, \gamma_{2}\right)-\phi_{1}\left(a_{1}, \beta_{1}, \gamma_{1}\right)-\phi_{8}\left(a_{3}, \beta_{2}, \gamma_{2}\right)}{\sqrt{ }\left\{\phi_{1}\left(\alpha_{1}, \beta_{1}, \gamma_{1}\right) \phi_{2}\left(\alpha_{2}, \beta_{2}, \gamma_{3}\right)\right\}} \\
& =\frac{-2 \Sigma \beta_{3}, \gamma_{3} \sin A+\left\{\Sigma\left(l_{1}+l_{2}\right) a_{2}\right\} \Sigma a_{3} \sin A}{\sqrt{ }\left\{\phi_{1}\left(a_{1}, \beta_{1}, \gamma_{1}\right\}\left\{\phi_{2}\left(a_{2}, \beta_{3}, \gamma_{2}\right)\right\}\right.} ;
\end{aligned}
$$

whence, by substituting the values for $a_{1}, \beta_{1}, \gamma_{1}, a_{2}, \beta_{2}, \gamma_{2}$, we obtain

$$
\cos \theta=\frac{-1-\Sigma l_{1} l_{3}+\Sigma \cos A\left(l_{1}+l_{1}+m_{1} n_{3}+m_{2} n_{1}\right)}{\sqrt{ }\left\{\left[1+\Sigma l_{1}^{2}-2 \Sigma \cos A\left(l_{1}+m_{1} n_{1}\right)\right]\left[1+\Sigma l_{2}^{2}-2 \Sigma \cos A\left(l_{2}+m_{8} n_{2}\right)\right]\right\}}
$$

Hence, if the circle $(l, m, n)$ make the same angle $\theta$ with each of the three circles

$$
(\sec A, 0,0), \quad(0, \sec B, 0), \quad(0,0, \sec \sigma),
$$

we nust have, by (7),

$$
\cos \theta \sqrt{ }\left[1+\Sigma l^{2}-2 \Sigma \cos A(l+m u)\right]
$$

$=\{-1-l \sec A+\cos A(l+\sec A)+\cos B(m+n \sec A)$

$$
+\cos C(n+m \sec A)\} / \sqrt{ }\left[1+\sec ^{3} A-2\right]
$$

$=\sec A\left\{-l\left(1-\cos ^{2} A\right)+m(\cos O+\cos A \cos B)\right.$

$$
+n(\cos B+\cos A \cos O)\} / \tan A
$$

$=\sec A\left\{-l \sin ^{2} A+m \sin A \sin B+u \sin A \sin C\right\} / \tan A$

$=-l \sin A+n \sin B+n \sin O$, and in like manner

$=+l \sin A-m \sin B+n \sin C$

$=+l \sin A+m \sin B-n \sin C$;

therefore $\quad l \sin A=m \sin B=n \sin O$. 
1889.] Mr. W. W. Taylor on some Rings of Circles, \&c. 403

Let each of these $=p$. Then, substituting for $l, m, n$ their values in terms of $p$,

$\cos \theta \sqrt{ }\left[1+p^{2} \Sigma \operatorname{cosec}^{2} A-2 p \Sigma \cot A-2 p^{2} \Sigma \cos A \operatorname{cosec} B \operatorname{cosec} O\right]=p$; or, putting $\cot A+\cot B+\cot C=\cot \omega$,

$$
\begin{gathered}
\cos \theta \sqrt{ }\left[1+p^{2} \operatorname{cosec}^{2} \omega-2 p \cot \omega-4 p^{2}\right]=p, \\
\cos \theta=\frac{p}{\sqrt{ }\left\{(1-p \cot \omega)^{2}-3 p^{2}\right\}}=\frac{1}{\sqrt{ }\left(\lambda^{3}-3\right)}, \\
\lambda=\frac{1}{p}-\cot \omega .
\end{gathered}
$$

Making these substitations, the equation of the circle $(l, m, n)$ reduces to the form

$$
\Sigma \beta \gamma \sin A-p(\Sigma a \operatorname{cosec} A)(\Sigma a \sin A)=0,
$$

or $\quad-p \Sigma a^{2}+\Sigma \beta \gamma \sin A-p \Sigma \beta \gamma \operatorname{cosec} B \operatorname{cosec} O\left(\sin ^{2} B+\sin ^{8} \theta\right)=0$,

or $\quad p \Sigma a^{2}-\Sigma(\beta \gamma \sin A(1-p \cot \omega)+p \Sigma \beta \gamma \cos A=0$,

[since $\frac{1}{2} \operatorname{cosec} A \operatorname{cosec} B \operatorname{cosec} C\left(\sin ^{2} A+\sin ^{2} B+\sin ^{2} C\right)$

$+\frac{1}{2} \operatorname{cosec} A \operatorname{cosec} B \operatorname{cosec} O\left(\sin ^{2} B+\sin ^{2} C-\sin ^{2} A\right)$

$$
=\cot \omega+\operatorname{cosec} A \cos A] \text {, }
$$

and substitating $\lambda$ for $(1-p \cot \omega) / p$, we obtain for the equation of our circle

$$
\Sigma a^{2}+\Sigma \beta \gamma \cos A-\lambda \Sigma \beta \gamma \sin A=0
$$

and, if this touch the circles $A_{1}, B_{1}, C_{1}$,

$$
\begin{gathered}
\sqrt{ }\left(\lambda^{2}-3\right)=\sec \theta=1, \\
\lambda= \pm 2 .
\end{gathered}
$$

The formulø for the centre of a circle become in this case

$$
\left.\begin{array}{l}
a=R(\sin A+\lambda \cos A) /(\lambda+\cot \omega) \\
\beta=R(\sin B+\lambda \cos B) /(\lambda+\cot \omega) \\
\gamma=R(\sin C+\lambda \cos C) /(\lambda+\cot \omega)
\end{array}\right\}
$$

With the same substitutions

$\rho^{8}=R^{2}\left(\Sigma l^{2}-2 \Sigma m i n \cos A-2 \Sigma l \cos A+1\right)$

$=R^{2}\left(\Sigma p^{2} \operatorname{cosec}^{2} A-2 \Sigma p^{2} \cos A \operatorname{cosec} B \operatorname{cosec} C-2 \Sigma p \cot A+1\right)$

$=R^{2}\left(p^{2} \operatorname{cosec}^{2} \omega-2 p^{2} \Sigma(1-\cot \Lambda \cot B)-2 p \cot \omega+1\right)$ $2 \mathrm{D} 2$ 
404 Mr. W. W. Taylor on some Rings of Circles, \&c. [June 13,

$$
\begin{aligned}
& =R^{2}\left(p^{2} \operatorname{cosec}^{2} \omega-4 p^{2}-2 p \cot \omega+1\right) \\
& =R^{2}(p \cot \omega-1)^{2}-3 p^{2} \\
& =R^{2}\left(\lambda^{2}-3\right) p^{2} \\
& =R^{2}\left(\lambda^{2}-3\right) /(\lambda+\cot \omega) \\
\rho & = \pm R \sqrt{ }\left(\lambda^{2}-3\right) /(\lambda+\cot \omega) \ldots \ldots \ldots . .
\end{aligned}
$$

The cosine of the angle between this circle (8) and a ring circle $(l, m, n)$ for which $l \cos A+m \cos B+n \cos C=1$, is

$-1-\Sigma l p \operatorname{cosec} A+\Sigma\{l \cos A+p \cot A+p l l(\cos B \operatorname{cosec} C+\cos C \operatorname{cosec} B)\}$

$$
\begin{aligned}
\overline{\sqrt{ }\left\{\left[\left(l^{2}+m^{2}+n^{2}\right)-(l \cos A+m \cos B+n \cos C)^{2}-2 \Sigma m n \cos A\right]\right.} \overline{\left.\left(\lambda^{2}-3\right) p^{2}\right\}} \\
=\frac{-1+\Sigma l p(\sin A-\cos A \cot \omega)+1+p \cot \omega}{p \sqrt{ }\left\{\left(\Sigma l^{2} \sin ^{2} A-2 \Sigma m n \sin B \sin C\right)\left(\lambda^{2}-3\right)\right\}} \\
\quad=\frac{\Sigma l \sin A}{\sqrt{ }\left\{\left(\Sigma l^{2} \sin ^{2} A-2 \Sigma m n \sin B \sin C\right)\left(\lambda^{2}-3\right)\right\}} \quad \ldots \ldots \ldots \ldots(11) .
\end{aligned}
$$

The series of circles included in the equation

$$
\Sigma \alpha^{2}+\Sigma \beta \gamma \cos A-\lambda \Sigma \beta \gamma \sin A=0
$$

has been discussed by Professor P. H. Schoute, in Vol. IIr., Series 3, of the Verslagen en Mededeelingen of the Koninklijke Aliademie van Wetenschappen, Amsterdam, from an entirely different point of view. He shows that, when a point $P$ moves so that, if $D, E, F$ be the feet of the perpendiculars from it on the sides of the triangle, the Brocard angle of the triangle $D E F$ is constant, the locus of $P$ is a circle of the above series, and $\lambda$ is the cotangent of the said Brocard angle.

He has shown that this series of circles includes, as particular cases: the Brocard circle $(\lambda=\cot \omega)$; the imaginary circle whose equation is

$$
\Sigma \alpha^{2}+\Sigma \beta \gamma \cos A=0, \quad(\lambda=0) ;
$$

the circle $A B C(\lambda=\infty)$; the Lemoine line $(\lambda=-\cot \omega)$, and the isodynamic points $(\lambda= \pm \sqrt{ } 3)$.

Of these six particular results the first three are obvious by a comparison of the equation No. 8 with the equations of the other circles, and the last three can be obtained by making the centre of the circle lie on the locus.

The condition for this is

$$
\left|\begin{array}{ccc}
2 & \cos C-\lambda \sin C & \cos B-\lambda \sin B \\
\cos C-\lambda \sin C & 2 & \cos A-\lambda \sin A \\
\cos B-\lambda \sin B & \cos A-\lambda \sin A & 2
\end{array}\right|=0
$$


which reduces to the form

$$
\left(\lambda^{2}-3\right)(\lambda \sin A \sin B \sin C+1+\cos A \cos B \cos C)=0 .
$$

The coordinates of the point circle, for which $\lambda=\sqrt{ } 3$, must be given by substituting this value in the equations of the centre of a circle (9).

Then

$$
\begin{aligned}
a & =R(\sin A+\sqrt{ } 3 \cos A) /(\sqrt{ } 3+\cot \omega) \\
& =2 R \cos \left(A-60^{\circ}\right) /(\sqrt{ } 3+\cot \omega), \\
\beta & =2 R \cos \left(B-60^{\circ}\right) /(\sqrt{ } 3+\cot \omega), \\
\gamma & =2 R\left(\cos C-60^{\circ}\right) /(\sqrt{ } 3+\cot \omega),
\end{aligned}
$$

and the remaining value

$$
\lambda \sin A \sin B \sin C+1+\cos A \cos B \cos C=0,
$$

or

$$
\lambda=-\cot \omega,
$$

gives all the coordinates of the centre infinite. This shows that the circle is a straight line which is at once fonnd to be

The centre of the circle

$$
\frac{a}{a}+\frac{\beta}{b}+\frac{\gamma}{c}=0
$$

$$
\Sigma(\beta \cos B-\gamma \cos C)^{2}+\Sigma(\beta \sin B+\gamma \sin C)^{2} \equiv \Sigma \alpha^{2}+\Sigma \beta \gamma \cos A=0
$$

is Lemoine's point $K$, and its radius is

$$
R \tan \omega \sqrt{ }(-3) \text {. }
$$

The equation of the real circle corresponding to this (centre $K$ radius $=R \tan \omega \sqrt{ } 3)$ is

$$
4 \Delta\left\{\Sigma \alpha^{2}+\Sigma / \beta \gamma \cos A\right\}-3 \tan \omega\{\Sigma a \alpha\}^{8}=0 .
$$

We will now for a time desert analysis and employ inversion, using inversion in the sense that we take a fixed point $O_{n}$, and find a point $Q$ corresponding to any other point $P$, such that $O_{n}, P, Q$ are in a straight line, and the rectangle $O_{n} P, O_{n} Q$ is equal to a constant (the square of the radius of inversion).

If we take the centre and radius of the circle $A B C$ as the centre and radius of inversion, the three-ring circles invert into themselves, and a circle cutting them at the angle $\theta$ must invert into a circle cutting them at the angle $\theta$; we see, therefore, that the $+\lambda$ circle of Schoute's series inverts into the $-\lambda$ circle of his series, and if $V, W$ be the isodynamic points, $O V . O W=R^{2}$. It also follows that $O$ 
is the external centre of similitude of the $\pm \lambda$ pair of Schoute's circles.

If, again, we take $O_{n}$ the centre of inversion on Lemoine's linethe radical axis of the system-and the tangent from $O_{n}$ to the circle $A B C$ as the radius of inversion, each circle of Schoute's system will invert into itself; but the three-ring circles will assume a different position for each position of $O_{n}$, and will always possess Schoute's system of circles each for each as before. Their points of contact will accordingly form new triangles, each of which possesses the same system of Schoute's circles. That these are the co-Brocardal triangles of $A B O$ can be proved by finding the envelope of a side as $O$, moves along Lemoine's line, or thus:-The three-ring circles of the co-Brocardal triangles must touch a pair of circles, which are coaxal with the Brocard circle and the circle $A B C$, and must be orthogonal to circle $A B O$; and the only rings of three circles that satisfy these conditions are the rings of circles obtained by our inversion.

This can also be proved thus. All our three-ring circles touch or cut all Schoute's circles at the same angle. So, taking $(l, m, n)$, ( $\sec A, 0,0)$ as two specimens of three-ring circles, we have, by (11),

$$
\Sigma l \sin A=\sqrt{ }\left\{\Sigma l^{2} \sin ^{2} A-2 \Sigma m n \sin B \sin C\right\},
$$

or

$$
\Sigma m n \sin B \sin C=0
$$

and any ring-circle has an cquation of the form

$$
l \sin A \cdot A_{1}+m \sin B \cdot B_{1}+n \sin C \cdot C_{1}=0 .
$$

This gives, as the form of the gcneral equation of a three-ring circle,

$$
\Lambda_{2} \equiv \Lambda_{1}-B_{1}(1+\mu)-C_{1}\left(1+\frac{1}{\mu}\right)=0 .
$$

The common chord of this circle and the circle $A B C$ is

$$
\mu \frac{a}{a}-\mu(1+\mu) \frac{\beta}{b}-(1+\mu) \frac{\gamma}{c}=0,
$$

and, as this is a quadratic equation in $\mu$, the envelope of all such lines is given by the equation

$$
\left(\frac{a}{a}-\frac{\beta}{b}-\frac{\gamma}{c}\right)^{2}=4 \frac{\beta \gamma}{b c},
$$

which is the equation of Brocard's cllipsc.

The circle $A_{3}$ meets the circle $A B C$ where

$$
A_{1} B_{1}+B_{1} C_{1}+C_{1} A_{1} \equiv\{\Sigma \beta \gamma \sin A\}^{2}=0,
$$


nud

$$
\begin{gathered}
A_{1}-B_{1}(1+\mu)-C_{1}\left(1+\frac{1}{\mu}\right)=0 ; \\
\mu A_{1}=-(\mu+1) B_{1}=C_{1}, \\
A_{1}=\mu B_{1}=-(\mu+1) C_{1}
\end{gathered}
$$

iat is, where

and where

The third point of this co-Brocardal triangle* must be

$$
-(\mu+1) A_{1}=B_{1}=\mu C_{1},
$$

and the equation to the other circles of the same ring will be

$$
\begin{aligned}
& B_{8} \equiv-A_{1}\left(1+\frac{1}{\mu}\right)+B_{1}-O_{1}(1+\mu)=0, \\
& C_{2} \equiv-A_{1}(1+\mu)-B_{1}\left(1+\frac{1}{\mu}\right)+O_{1}=0 .
\end{aligned}
$$

Here $A_{2}, B_{9}, C_{2}$ satisfy the relations

$$
\begin{gathered}
A_{2}+B_{2}+C_{2} \equiv-\left(\mu+1+\frac{1}{\mu}\right)\left(A_{1}+B_{1}+C_{1}\right) \\
B_{2} C_{8}+C_{2} A_{3}+A_{2} B_{8} \equiv\left(\mu+1+\frac{1}{\mu}\right)^{2}\left(B_{1} C_{1}+C_{1} A_{1}+A_{1} B_{1}\right) .
\end{gathered}
$$

Again, we will invert with regard to one of the isodynamic points $V, W$ as our centre of inversion, and the tangent to the circle $A B C$ as our radius of inversion. Since

$$
\begin{gathered}
O V . O W=R^{2}, \\
W V . W O=W O^{3}-R^{3}=W \eta^{2} ;
\end{gathered}
$$

therefore, inverting with respect to $W$, as above, $V$ inverts into the centre of the circle $A B C$, and the circle $A B C$ inverts into itsclf ; and two circles of the coaval system having become concentric hy inversion, the rest must have done so also, and the whole system of Schoute's circles bccomes a concentric system, and, in consequence of these circles having become concentric, the threc-ring circles become necessarily equal circles, and their points of contact form an equi-

- The coordinates of tho angular points of this triangle are given by the equations

$$
\begin{aligned}
& \frac{a}{a}=\mu \frac{\beta}{b}=-(\mu+1) \frac{\gamma}{c}, \\
& -(\mu+1) \frac{a}{a}=\frac{\beta}{b}=\mu \frac{\gamma}{c}, \\
& \mu \frac{a}{a}=-(\mu+1) \frac{\beta}{b}=\frac{\gamma}{a} .
\end{aligned}
$$


408 Mr. W. W. Taylor on some Rings of Circles, \&c. [June 13

lateral triangle. This applies not only to our original triangle, but to any of the co-Brocardal system of triangles; and as at the same time the other isodynamic point inverts into the centre of the circle $A B C$, the circles of Apollonius, which passed through both points $V, W$ and the angular points of the triangle, become straight lines passing through $O$ and the angular points of the equilateral triangles, that is to say, become diameters of the circle $A B C$ through the angular points of the equilateral triangles. Therefore in any triangle the circles of Apollonius cut one another at an angle of $60^{\circ}$.

The circle of inversion in this case is at the isodynamic point-in other words, is concentric with the circle

$$
\boldsymbol{\alpha}^{3}+\beta^{2}+\gamma^{2}+\Sigma \beta \gamma(\cos A \pm \sqrt{ } 3 \sin A)=0 .
$$

[The - sign gives the inner point $(V)$, the + sign the outer point $(W)$ ]. A circle concentric with this must have an equation of the form

$$
\Sigma a^{2}+\Sigma \beta \gamma(\cos A \pm \sqrt{ } 3 \sin A)+h(\Sigma a \sin A)^{2}=0 .
$$

If this be also of the form (4),

$$
\begin{aligned}
& l \sin A=1+h \sin ^{2} A, \\
& m \sin B=1+h \sin ^{2} B, \\
& n \sin C=1+h \sin ^{2} C,
\end{aligned}
$$

$-l \sin A \cos A+m \sin B \cos A+n \sin C \cos A$

$$
=\cos A \pm \sqrt{ } 3 \sin A+2 h \sin B \sin C,
$$

and also from above $=\cos A\left[1+h\left(-\sin ^{2} A+\sin ^{2} B+\sin ^{4} C\right)\right]$;

whence

$h\left[\cos A\left(\sin ^{9} A-\sin ^{2} B-\sin ^{2} C\right)+2 \sin B \sin C\right]=\mp \sqrt{ } 3 \sin A$,

or

$$
h=\frac{\mp \sqrt{ } 3}{2 \sin A \sin B \sin C},
$$

and the equation of the required circle becomes

$$
\Sigma a^{8}+\Sigma / 3 \gamma(\cos A \pm \sqrt{ } 3 \sin A) \mp \frac{\sqrt{ } 3}{2 \sin A \sin B \sin C}(\Sigma a \sin A)^{2}=0 .
$$

The upper signs will give an imaginary and the lower a real circle, 
showing that the inversion is in the first case across the point, in the second away from it.

The centre of the circle

$$
a^{2}+\beta^{2}+\gamma^{2}+\Sigma \beta \gamma \cos A=0
$$

was found to be the symmedian point $K_{1}$, and its radius

$$
\rho=R \tan \omega \sqrt{ }(-3) \text {. }
$$

Again, if we draw the chord $A K A^{\prime}$ of the circle $A B C$, the product $A K . K A^{\prime}$ is equal to

$$
R^{2}-O K^{2}=R^{2}-4 \rho^{2}
$$

(where $\rho^{\prime}$ is the radius of the Brocard circle, i.e., of the Schoute circle for which $\lambda=\cot \omega)=R^{2}-R^{2}\left(1-3 \tan ^{2} \omega\right)$, by (10),

$$
=3 R^{2} \tan ^{2} \omega \text {. }
$$

This proves that, if we invert with $K$ as centre of inversion, and the radius of this impossible circle as the radius of inversion, the circle $A B C$ will invert into itself ; consequently, all circles orthogonal to it will invert into circles orthogonal to it, aud the three-ring circles of the triangle $A B C$ will invert into the three-ring circles of the cosymmedian triangle; as this is a co-Brocardal triangle, the system of Schoute's circles must invert into one another as circles cut one another at the same angles as their inverses. It also follows that $K$ is the internal centre of similitude of each pair $( \pm \lambda)$ of Schoute's circles.

We can by means of this result obtain the equations of the six-ring circles which are orthogonal to the circle $A B C$, and are cut at equal angles by each of the Schoute circles. For, drawing the chords $A K A^{\prime}, B K B^{\prime}, C K C^{\prime}$ of the circle $A B C, A^{\prime}, B^{\prime}, C^{\prime}$ must, by our last result, be the points where the six-ring circles at $A B C$ meet the circle $A B C$ again ; and as the coordinates of $K$ are $a: b: c$, the equation of $A^{\prime} K$ is

$$
\beta \sin C=\gamma \sin B,
$$

and the equations of $A^{\prime} B, A^{\prime} C$ are found by eliminating $\beta$ and $\gamma$ from the equations of $A^{\prime} K$ and the circle $A B C$; therefore the equation of $A^{\prime} B$ is

$$
2 a \sin C+\gamma \cdot \sin A=0 \text {; }
$$

and therefore, by equation (4), a ring-circle of the six-ring through $A^{\prime} B$ will bave for its equation

$$
2 A_{1}+U_{1}=0,
$$


and the equations of a complete ring of the six-ring circles will be

$$
\begin{aligned}
& B_{1}+2 C_{1}=0, \\
& A_{1}+2 O_{1}=0, \\
& O_{1}+2 A_{1}=0, \\
& B_{1}+2 A_{1}=0, \\
& A_{1}+2 B_{1}=0, \\
& C_{1}+2 B_{1}=0 .
\end{aligned}
$$

The value of $\lambda$ for the Schoute circles that touch the six-ring circles is

$$
\lambda= \pm 2 \sqrt{ } 3 \text {. }
$$

The general equation of a member of the six-ring circles can be found by taking an arbitrary point $O_{n}$ on Lemoine's line, drawing the lines $O_{n} A, O_{n} B^{\prime}$, and finding where these meet the circle $A B C$ again, and forming the equation of that chord of the circle $A B C$; whereupon we at once know the equation of the corresponding ring-circle, and proceeding in the same way we can find the equations of all the circles of any six-ring system.

We will briefly indicate how the same can be done for any other ring. The chords common to the circles of the ring and the circumscribed circle form a harmonic polygon (Cnscy's "Sequel to Euclid," 199-20(ن), and always touch an ellipse of a family of which Brocard's is the best known example. Their centres lie on another ellipse whose foci are the centres of $a \pm \lambda$ pair of Schoute's circles.

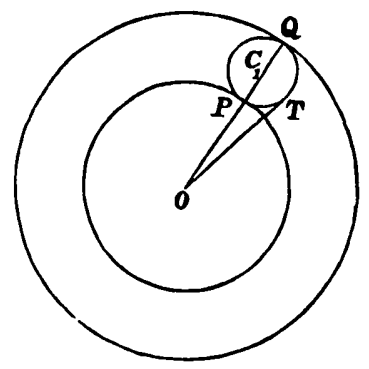

To determine the valne of $\lambda$ for the tangent circle to any ring, formula (11) shows us that the angle at which any of Schoute's circles cuts the three-ring circles depends only on the value of $\lambda$, and not on the angles $A, B, C$; so we can determine the value of $\lambda$ for an equilateral triangle. 
1889.] Mr. W. W. Taylor on some Rings of Oircles, \&c.

Now, for any ring of $r$ ciroles, we must have in the above figarewhere $\mathrm{O}_{2}$ is the centre of $P T Q$ one of the ring-circles, and $P, T, Q$ are the points where the ring-cirole meets the $\lambda$-Schoute circle, the circle $A B O$, and the $-\lambda$-Schoate circle-

$$
\tan \frac{\pi}{r}=\tan O_{1} O T=O_{1} T / \sqrt{ }(O P . O Q)=\frac{1}{2}(O Q-O P) / \sqrt{ }(O P . O Q),
$$

and therefore, taking the values of $O P, O Q$ from equation (10) with due regard to sign, and remembering that $\lambda<\sqrt{ } 3$ numerically, and

$$
\cot \omega=\sqrt{ } 3,
$$

we have $\tan \frac{\pi}{r}=\frac{1}{2}\left(\frac{1}{\lambda-\cot \omega}-\frac{1}{\lambda+\cot \omega}\right) / \sqrt{ }\left(\frac{1}{\lambda^{8}-\cot ^{3} \omega}\right)$

$$
\begin{array}{r}
=\frac{\cot \omega}{\sqrt{ }\left(\lambda^{3}-\cot ^{2} \omega\right)}=\frac{\sqrt{ } 3}{\sqrt{ }\left(\lambda^{3}-3\right)}, \\
\lambda^{2}=3 \operatorname{cosec}^{3} \frac{\pi}{r}, \\
\lambda= \pm \sqrt{ } 3 \operatorname{cosec} \frac{\pi}{r},
\end{array}
$$

and for the six-ring circle

$$
\lambda= \pm 2 \sqrt{ } 3 .
$$

The general relation between $l, m, n$, when a circle belongs to the ring of $r$ circles, is found by substituting the values

$$
\cos \theta=1 \text { and } \lambda= \pm \sqrt{ } 3 \operatorname{cosec} \frac{\pi}{r}
$$

from above in formula (11), and can be written

$$
(\Sigma l \sin A) \tan \frac{\pi}{r}=\sqrt{ } 3 \cdot \sqrt{ }\left(\Sigma l^{2} \sin ^{2} A-2 \Sigma m n \sin B \sin \sigma\right)
$$

and this is, consequently, also the relation between $l, m, n$ when

$$
l a+m \beta+n \gamma=0
$$

is a side of an harmonic polygon of $r$ sides belonging to the same system.

The locus of the centres of the ring-circles of any series is plainly an ellipse or other conio of which the foci are the centres of the $\pm \lambda$ circles that touch all the members of that ring.

For, if $O_{1}, O_{2}$ be the centres of the $\pm \lambda$ circles that touch at $P$ and 
412 Mr. W. . Taylor on some Rings of Circles, \&c. [June 13, $Q$ the ring-circle whose centre is $C_{1}$, then

$$
\begin{aligned}
O_{1} C_{1}+O_{1} O_{2} & =R_{1}-C_{1} P+R_{8}+C_{1} Q=R_{1}+R_{8} \\
& =\text { a constant }=2 \lambda R \sqrt{ }\left(\lambda^{2}-3\right) /\left(\lambda^{2}-\cot ^{2} \omega\right) .
\end{aligned}
$$

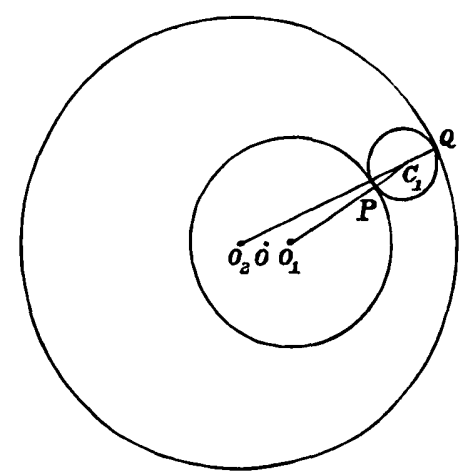

However, we can find the equation of this locus more readily by finding the locus of the pole of

$$
l a+m \beta+n \gamma=0,
$$

subject to the condition (12), which may be written in the form

$$
(\Sigma l \sin A)^{2}\left(\tan ^{2} \frac{\pi}{r}+3\right)=6 \Sigma l^{2} \sin ^{2} A .
$$

Since $a, \beta, \gamma$, a point on the locus, is the pole of

$$
l a+m \beta+n \gamma=0
$$

with respect to the circle $A B C$,

$$
\frac{l}{b \gamma+c \beta}=\frac{m}{a \gamma+c \alpha}=\frac{n}{a, \beta+b a},
$$

and the locus of the pole must therefore be given by the equation

$$
\begin{gathered}
\{\Sigma(a b \gamma+a c \beta)\}^{2}\left(\tan ^{2} \frac{\pi}{r}+3\right)=6 \Sigma(a b \gamma+a c \beta)^{2}, \\
(2 \Sigma b c a)^{2}\left(\tan ^{2} \frac{\pi}{r}+3\right)=12 \Sigma\left(b^{3} c^{2} a^{2}+a^{2} b c c^{2} \gamma\right), \\
\Sigma\left(l^{2} c^{2} a^{2}+2 a^{2} b c \mid 3 \gamma\right)\left(\tan ^{2} \frac{\pi}{r}+3\right)=3 \Sigma\left(b^{2} c^{2} \alpha^{2}+a^{2} b c \mid \beta \gamma\right), \\
\Sigma b^{2} c^{2} \alpha^{2} \tan ^{2} \frac{\pi}{r}+\Sigma a^{2} b c \beta \gamma\left(2 \tan ^{2} \frac{\pi}{r}+3\right)=0 .
\end{gathered}
$$


1889.] Mr. W. W. Taylor on some Rings of Circles, \&c.

It is plain that the sides also of the harmonic polygons, being the polars of these centres with respect to the circle $A B C$, must envelope an ellipse.

Its equation, being the envelope of

$$
l a+m \beta+n \gamma=0
$$

subject to the condition (12), is

$$
\Sigma b^{2} c^{2} \alpha^{2} \tan ^{2} \frac{\pi}{r}-\left(\tan ^{2} \frac{\pi}{r}+3\right) \Sigma a^{2} b c \beta \gamma=0 .
$$

Both series of ellipses belong to the same family

$$
\Sigma b^{2} c^{2} a^{2}+\mu \Sigma a^{2} b c \beta \gamma=0
$$

and the values of $\mu$ for a pair of them are connected by the relation

$$
\mu_{1}+\mu_{2}=1 \text {. }
$$

The usual formulø for the foci of the conic

$$
A \alpha^{2}+B \beta^{2}+C \gamma^{2}+2 D \beta \gamma+2 E \gamma \alpha+2 F a \beta=0
$$

are $a^{2}\left(A^{\prime} a^{2}+B^{\prime} b^{2}+C^{\prime} c^{2}+2 D^{\prime} b c+2 E^{\prime} c a+2 F^{\prime} a b\right)$

$$
-4 \Delta a\left(b F^{\prime}+c E^{\prime}+a A^{\prime}\right)+4 \Delta^{2} A^{\prime}
$$

$=$ two like expressions in $\beta$ and $\gamma$, where $A^{\prime}, B^{\prime}$, \&c. are the minors of $A, B, \& c$. in the determinant

$$
\left|\begin{array}{lll}
A & F & E \\
F & B & D \\
E & D & C
\end{array}\right| \text { (Whitworth, p. 269) }
$$

In the present case these equations reduce to

$$
\begin{aligned}
a^{2}\left\{\mu\left(-\Sigma a^{4}+2 \Sigma b^{2} c^{2}\right)-2 \Sigma a^{4}\right\} \\
-4 \Delta a a\left\{\left(b^{2}+c^{2}-a^{2}\right) \mu-2 a^{2}\right\}-4 \Delta^{2} a^{2}(\mu+2)=\& c .
\end{aligned}
$$

Patting each of these $=K$, and rearranging,

$$
\begin{gathered}
a^{2}\left\{\mu\left(\Sigma a^{2}\right)^{2}-2(\mu+1) \Sigma a^{4}\right\}-4 \Delta a a\left\{\mu \Sigma a^{2}-2(\mu+1) a^{2}\right\} \\
+4 \Delta^{2} a^{2}\{\mu-2(\mu+1)\}=K,
\end{gathered}
$$

and two like equations.

Eliminating from these the ratios

$$
\mu:-2(\mu+1): K,
$$


414 Mr. W. W. Taylor on some Rings of Circles, \&c. [June 13, we obtain, as the equation of the locus of the foci,

$$
\left|\begin{array}{lll}
\left(a \Sigma a^{2}-2 \Delta a\right)^{2}, & a^{2} \Sigma a^{4}-4 \Delta a^{3} a+4 \Delta^{2} a^{2}, & 1 \\
\left(\beta \Sigma a^{2}-2 \Delta b\right)^{2}, & \beta^{2} \Sigma a^{4}-4 \Delta b^{3} \beta+4 \Delta^{2} b^{2}, & 1 \\
\left(\gamma \Sigma a^{3}-2 \Delta c\right)^{2}, & \gamma^{2} \Sigma a^{4}-4 \Delta c^{3} \gamma+4 \Delta^{2} c^{2}, & 1
\end{array}\right|=0,
$$

which, on expansion, yields the two factors

$$
\Sigma b c\left(b^{2}-c^{3}\right) a \text { and } a b c \Sigma a^{2}-\Sigma a^{8} \beta \gamma \text {. }
$$

For the locus the real foci are on the straight line $K O$,

$$
\Sigma b c\left(b^{2}-c^{2}\right) a=0,
$$

and the imaginary ones on the Brocard circle

$$
a b c \Sigma a^{2}=\Sigma a^{8} \beta \gamma \text {. }
$$

Since the equation (13) can be arranged in the form

$$
(\Sigma b c a)^{2}+\overline{\mu-2} \Sigma a^{2} b c \hat{\gamma} \gamma=0 \text {, }
$$

the family of ellipses (13) have imaginary double contact with one another and the circle $A B C$ where they meet the line

$$
\Sigma b c a=0 .
$$

There is one parabola belonging to the series, which is the locus of centres of circles that touch Brocard's circle and Lemoine's line, and then

$$
\mu=\tan ^{2} \omega-3 \text {. }
$$

(Lemoine's line) ${ }^{2}$ is given twice over by making the discriminant vanish, and we also obtain Lemoine's point as a particular case of these conics.

To find the general equation of a circle of Apollonius, we know that it is orthogonal to the circle $A B C$, and therefore its equation is of the form (4),

$$
l \sin A \cdot A_{1}+m \sin B \cdot B_{1}+n \sin O \cdot O_{1}=0 .
$$

To be a circle of Apollonius it must also pass through the point

$$
\sin \left(A+60^{\circ}\right): \sin \left(B+60^{\circ}\right): \sin \left(C+60^{\circ}\right) \text {. }
$$

Making these substitutions for $a, \beta, \gamma$ in $A_{1}$, we obtain, for

$$
\begin{aligned}
& a^{3}-\beta \gamma \cos A+\gamma a \cos B+\alpha \beta \cos C, \\
& \text { t }\left\{\sin ^{2} A+3 \cos ^{2} A-\cos A(\sin B \sin C+3 \cos B \cos C)\right. \\
& +\cos B(\sin A \sin C+3 \cos A \cos C) \\
& +\cos O(\sin A \sin B+3 \cos A \cos B)\} \\
& +\frac{1}{4} \sqrt{ } 3\{2 \sin A \cos A-\sin A \cos A+\sin B \cos B+\sin C \cos C\} \\
& =\frac{1}{2}\{1+\cos A \cos B \cos C+\sqrt{ } 3 \sin A \sin B \sin C\} ;
\end{aligned}
$$


1889.] Mr. W. W. Taylor on some Rings of Circles, \&c.

therefore, in this case, $A_{1}=B_{1}=C_{1}$,

and the necessary condition for the above equation (4) to represent a circle of Apollonius is

$$
l \sin A+m \sin B+n \sin C=0 .
$$

The particular one through the angular point $A$ of the original triangle must satisfy (4), when we put

$$
a: \beta: \gamma=1: 0: 0 ;
$$

$A_{1}$ becomes 1,

$$
B_{1}=O_{1}=0
$$

therefore

$$
\imath=0 \text {, }
$$

and the equation reduces to $B_{1}=O_{1}$.

Hence

$$
B_{1}=O_{1}, \quad O_{1}=A_{1}, \quad A_{1}=B_{1}
$$

are the three primary circles of Apollonius. Any other can be found by making (4) pass through some other point on the circle $A B C$. The circle on $V W$ as diameter is plainly the smallest of all these circles, and its equation can be found by making its centre lie on $O K$ whose equation is

$$
\Sigma a\left(b^{2}-c^{2}\right) a=0,
$$

or, more simply, by making the common chord with the circle $A B C$,

$$
l a+m \beta+n \gamma=0,
$$

parallel to Lemoine's line. The condition for this is

$$
\left|\begin{array}{ccc}
l, & m, & n \\
\frac{1}{a}, & \frac{1}{b}, & \frac{1}{c} \\
a, & b, & c
\end{array}\right|=0,
$$

or

$$
l a\left(b^{8}-c^{2}\right)+m b\left(c^{2}-a^{2}\right)+n c\left(a^{9}-b^{2}\right)=0 .
$$

And the equation to this circle may be obtained by eliminating $l, m, n$ from the three equations

$l \sin A \cdot A_{1}+m \sin B \cdot B_{1}+n \sin O . O_{1}=0$,

$l \sin A+m \sin B+n \sin O=0$,

$l \sin A\left(\sin ^{2} B-\sin ^{2} C\right)+m \sin B\left(\sin ^{2} C-\sin ^{2} A\right)$

$$
+n \sin O\left(\sin ^{2} A-\sin ^{2} B\right)=0 \text {. }
$$


416 Mr. W. W. Taylor on some Rings of Circles, \&c. [June 13, Its equation, therefore, is

$$
\left|\begin{array}{ccc}
A_{1}, & B_{1}, & C_{1} \\
1, & 1, & 1 \\
\sin ^{2} B-\sin ^{2} C, & \sin ^{2} C-\sin ^{2} A, & \sin ^{2} A-\sin ^{3} B
\end{array}\right|=0,
$$

or $\quad A_{1}\left(2 a^{3}-b^{2}-c^{2}\right)+B_{1}\left(2 b^{2}-a^{2}-c^{2}\right)+C_{1}\left(2 c^{2}-a^{2}-b^{2}\right)=0$.

We bave previously omitted to remark that, if we take any other point anywhere, and invert with the tangent to the circle $A B O$ as the radius of inversion, we shall obtain i new set of harmonic polygons, ring-circles and Schoute's circles; as is evident, since all curves cut at the same angle as their inverses.

It is worthy of notice that the circles $A B C, B C W, C A W$, and $A B W$, being the inverses of the eircle $A B C$, and of the sides of an equilateral triangle, intersect at angles of $60^{\circ}$, and the circles of Apollonius round $V A W, V B W, V C W$, being the inverses of the bisectors of the angles of the equilateral triangle, intersect at $60^{\circ}$ and bisect the angles between the circles $B O W, O A W, A B W$.

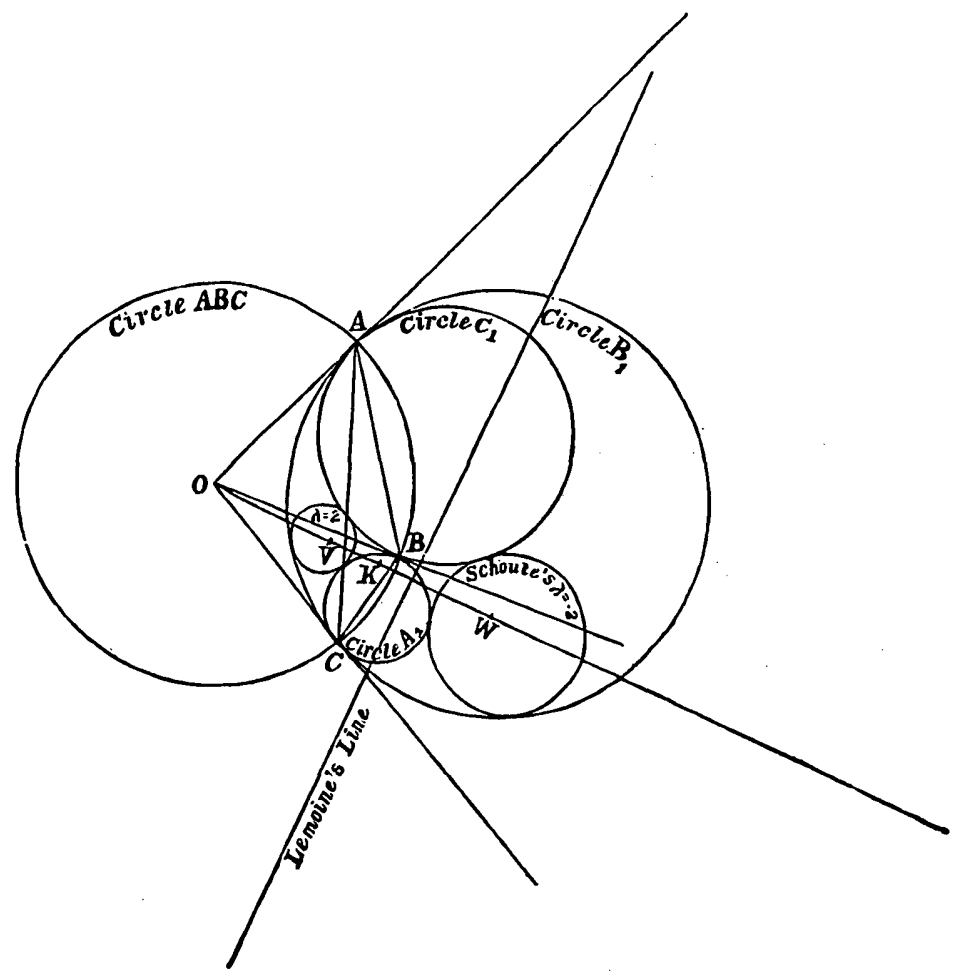




\section{Presents received during the Recess :-}

"Educational Times," for July-October, 1889.

"Tho Scicntific Transactious of the Royal Dublin Socicty," Vol. IV., Purto I. to v.

"I'ho Sciontific Proccedings of the Royal Dublin Society," Vol. vi., Parts III. to vi.

"Mcmoirs of tho National Academy of Sciences," Vol. rv., Part I. ; Washington, 1888.

"Annals of Mathematics," Vol..1v., No. 6; Vol. v., No. 1 ; Virginia, 1889.

"Bulletin des Sciencos Mathćmatiques," 'Iorne xur., June to Septomber, 1889.

"Bulletin do la Sociétó Mithématique de France," 'Tome xvır., Nos. 2, 3.

"Beiblüttor zu don Annalen der Physik und Chemio," Band xir,, Stücke 5-9.

"Jahrbuch über diu Fortschritto der Mathematik," Band xvirr., Ileft 3.

"Atti della Reule Accitdemin dei Lincei-Rendiconti," Vol. v., Fasc. 4 to 12.

"Atti dolla Ir. Accadomia dui Lincoi, Momorio dolla Classo di Scicnzo Fisiche, Mathematiche e Naturali," Vols. III. and Iv.

"Vicrteljahrschrift dor Naturforschenden Gesellschaft in Zürich," Jahr. xxxır., Hefte 3 and 4 ; xxxiv., Hoft 1.

"Bollettino delle Pubblicazioni Italiano riccvuto per Diritto di Stampa," Nos. 83-90.

"Archives Néprlandaises des Sciences Exactes et Naturelles," Tome xxirr., Livr. 3 and 4.

"Sitzungsberichte dor Königlich-Proussischen Akademic dor Wissenschaften zu Berlin," 1889, I. to xxI.

"Atti del Realo Istituto Vencto," 'T'omo vi., Disp. 10; 'Tomo vir., Disp. 1 and 2.

"Jornal des Scienciay Drathernaticas o Astronomicas," Vol. Ix., No. 2.

"Memorias de la Sociedad Cientifica- Antonio Alzate," 'T'omo II., Nos. 8 to 10 .

" Rendiconti del Circolo Matematico di Palermo," Fasc. 1n.-v.

"The Mathernutical 'l'heory of Electricity and Magnetism," by H. W. Watson, Sc.D., F.R.S., and S. II. Burbury, M.A., Vol. II. "Magnetism and Electrodynamics," Oxford, Clarendon l'ress, 1889.

"Amorican Journal of Mathematicy," Vol. xi., No. 4; Vol. xir., No. 1; Baltimore.

"Proceerlings of the Royal suciety," Nos. 280, 281, and 282.

"Momoirs and Procecding's of tho Manchuster Litcrary and Philosophical Socicty," Vol. II., Fourth Sorics, 8vo; Manchester, 1889.

"A 'Treatiso on Analytical Mechanics," by Bartholomew Price, M.A., F.R.S.;

Vol. II., "Dynamics of a Material Systom," Sccond Ldition, 8vo; Oxford, 1889.

"Transactions of the Royal Irish Acadcmy," Vol. xxix., Parts vi. to xI.

"Smithsonian Report," 1886, Part I., 8vo; Washington, 1889.

"Acta MIathernatica," xII., 3 and 4.

"Annali di Matematica," Tomo xvir., Fasc. 2.

" QEuvres complètes do Christiaan Huygens," Tomo ir., 4to ; La Hayo, 1889.

"Bulletins de l'Acadérnic Royale de Bolgique," 3me Serie, T. xIv. to xvir., 1887, 88, 89; Annuaire, 1888, 1889.

" Journal für dio reino und angowandto Muthcmatik," Band 105, Hefte I. and II.

“Journal de l'École Polytechnique," Cuhicr 58 ; Paris, 1889.

VOL. XX. -NO. 369 . 
" Sitzungsberichte der Physikalisch-medicinischen Societät in Erlangen," 1888; Munchen, 1889.

"Journal of the College of Science, Imperial University, Japan," Vol. III., Parts I. and Ir.

Pamphlets by M. Maurice d'Ocagne :-

"Sur certaines Courbes qu'on peut adjoindre aux Courbes Planes pour l'étude de leurs Propriétés Infinitćsimales." (Annerican Jour. of Math., Vol. XI., No. 1.)

"Calcul direct des Termes d'une réduite de rang quelconque d'uno Fraction Continue Périodique."

"Détormination du Rayon de Courbure de la Courbe Intégrale." (Nouvclles Annales.)

"Quelques propriétés de l'Ellipse ; Doviation, Ecart normal." (Nouvellcs Annalcs.)

"Sur les Systèmes de Péninvariants principaux d'une Forme Binaire. (Bulletin de la S. Hath. de France.)

"Formules nouvelles pour résoudro lo problème de la Carte au moyen de données particulic̀res." (Revue Mraritime et Coloniale, Fev., 1889.)

\section{A P P E N D IX.}

Mr. Basset points out that the following corrections should be supplied in his investigation of the stability of Maclaurin's Spheroid (Vol. xIX., pp. 52-54):-“"The correct result, which was first obtained by Riemamn, is that for an ellipsoidal displacement, the spheroid becomes unstable, whon the excentricity exceeds the root of the equation

$$
e\left(1-e^{2}\right)^{4}\left(3+4 e^{9}\right)=\left(3+2 e^{2}-4 e^{4}\right) \sin ^{-1} e,
$$

which gives $e$ equal to about 95. ." See his work on "Hydrodynamics," Vol. II., p. 124.

The following recently published papers bear upon the subject :Love, Phil. Mag., Vol. xxviI., p. 254.

Bryan, Phil. Trans., 1889, p. 187, and Proc. Oamb. Phil. Soc., Vol. vı., p. 248.

Thore is a paper by H. Weber in the Math. Annalen, Band xxxirr., Heft. 3, p. 391, "Zur complexen Multiplication elliptischen Funktionen." (See Prof. Greenhill's paper on "Complex Multiplication Moduli of Elliptic Functions," Vol. xIx., p. 362.) 